\title{
Civilisations
}

Revue internationale d'anthropologie et de sciences

humaines

$61-2 \mid 2013$

Chamanismes en mouvement

\section{Les nouveaux yajeceros urbains}

Une approche de la consommation rituelle du yajé en Colombie

\section{Alhena Caicedo-Fernández}

\section{(2) OpenEdition}

1 Journals

\section{Édition électronique}

URL : http://journals.openedition.org/civilisations/3239

DOI : $10.4000 /$ civilisations.3239

ISSN : 2032-0442

Éditeur

Institut de sociologie de l'Université Libre de Bruxelles

\section{Édition imprimée}

Date de publication : 28 juin 2013

Pagination : 53-67

ISSN : 0009-8140

\section{Référence électronique}

Alhena Caicedo-Fernández, «Les nouveaux yajeceros urbains », Civilisations [En ligne], 61-2 | 2013, mis en ligne le 14 juin 2016, consulté le 01 mai 2019. URL : http://journals.openedition.org/ civilisations/3239; DOI : 10.4000/civilisations.3239 


\title{
Les nouveaux yajeceros urbains \\ Une approche de la consommation rituelle du yajé en Colombie
}

\author{
Alhena CAICEDO-FERNÁNDEZ
}

Résumé : Depuis quelques années, dans plusieurs villes de Colombie, on observe une dissémination des pratiques rituelles associées à la prise de ayahuasca ou yajé, un psychotrope dont l'usage traditionnel, à des fins thérapeutiques et d'initiation chamanique, est propre aux paysans autochtones et métisses des contreforts de l'Amazonie. Aujourd'hui, ces pratiques engagent des personnes des classes moyennes et de l'élite, accédant à ces espaces pour différents motifs où le thérapeutique et le spirituel prédominent. S'offrant sous l'étiquette de " médecine traditionnelle ", la consommation rituelle de yajé est promue comme une pratique légitime et légale, sous la nouvelle modalité de spécialistes (les nouveaux taitas yajeceros) qui articulent les usages traditionnels autochtones du psychotrope avec les attentes du public urbain. Cette réflexion propose quelques explorations sur les nouveaux lieux du yajé en Colombie et montre quelques-unes de leurs particularités à travers le cas d'un nouveau taita yajecero et de sa fondation.

Mots-clés : yajé (ayahuasca), médecine traditionnelle indigène, néochamanisme.

Abstract: For several years, many Columbian cities have witnessed a rise of ritual practices linked to the ingestion of ayahuasca or yajé. The traditional use of this psychoactive substance is related to shamanic healing and initiation, as performed by the indigenous and mixed-blood peasants of the Amazonian foothills. Today, these practices also concern middle and high class individuals, motivated by therapeutic and spiritual motives predominantly. Presented under the label of "traditional medicine», the ritual intake of yajé is promoted as a legitimate and legal practice. A new class of experts (the new taitas yajeceros) articulate the traditional indigenous uses of this psychoactive substance and the expectations of an urban audience. This article explores some new places of yajés consumption in Columbia and discusses some of their particularities through the case study of a new taita yajecero and of his foundation.

Keywords: Yajé (ayahuasca), traditional indigenous medicine, neo-shamanism. 
$\mathbf{B}_{\text {la production et l'usage de drogues se soient intensifiées dans le monde entier }}$ l'usage des substances psychotropes a atteint de nos jours une croissance particulière dans de nouveaux contextes. L'ayahuasca ${ }^{1}$, psychotrope d'origine amazonienne, est l'une de ces substances dont la visibilité s'est accrue tant en Amérique latine qu'en Europe, en gagnant toujours plus d'adeptes. Lié à l'expansion de nouvelles Églises comme le Santo Daime ou l'UDV (Uniâo do Vegetal), à des pratiques inspirées des chamanismes traditionnels et au phénomène des thérapies alternatives, l'usage rituel de l'ayahuasca est une pratique émergente en expansion dans plusieurs villes latinoaméricaines. En Colombie, l'usage de ce psychotrope, mieux connu sous le nom de yajé, s'est disséminé dans l'espace urbain durant ces vingt dernières années.

Le yajé est une boisson psychotrope employée traditionnellement, en Colombie, par des populations indigènes (Ingas, Kamentsás, Sionas, Kofánes, Koreguajes, etc.) et par des métis du piémont de l'Amazonie (région du Putumayo-Caquetá), à des fins thérapeutiques et d'initiation chamanique. C'est pourquoi son usage a été lié aux populations paysannes indigènes et métisses du sud-ouest du pays, et aux secteurs populaires constitués d'immigrants de la campagne à la ville. Ces secteurs de la population ont recouru traditionnellement aux curanderos utilisant le yajé pour résoudre certains types de maladie ou de malheur. Si le yajé est de fait présent depuis longtemps en ville, on observe depuis peu la dissémination de sa consommation dans des secteurs de l'élite et de la classe moyenne, c'est-à-dire dans des secteurs de la société où ce psychotrope était pratiquement inconnu, ou associé à des croyances et à des superstitions attribuées aux classes populaires.

Ce processus d'urbanisation et d'élitisation de la prise de yajé ne peut se comprendre que par référence au contexte sociopolitique de la Colombie de la fin du $20^{\text {ème }}$ siècle. La réforme constitutionnelle de 1991 a marqué une étape importante dans la transformation de l'État colombien. Précédée d'une mobilisation sociale durant la décennie antérieure, la nouvelle constitution a voulu établir les bases d'un État plus décentralisé et participatif reconnaissant la nation colombienne dans sa diversité ethnique et culturelle ${ }^{2}$. Ainsi, au nom de la nouvelle charte constitutionnelle, l'État commence à reconnaître des droits culturels collectifs aux " groupes ethniques »: droits territoriaux, affectation de ressources, politiques différentielles de santé et d'éducation, représentation parlementaire, justice propre, etc. L'ethnicité devient ainsi un marqueur de différence sanctionné par l'État, qui détermine les signes diacritiques de ce qui peut ou ne peut pas être reconnu comme ethnique. En très peu de temps, les représentations sociales de l'indianité ont commencé à inverser leur valeur: on ne peut plus parler d'indiens mais bien d'indigènes. Les considérations historiques méprisantes sur l'indianité commencent à être revues dans de nombreuses instances, grâce à l'intervention d'universitaires et d'experts, tout ceci en suivant les nouveaux réquisits gouvernementaux et en s'appuyant sur la visibilité dans les médias, notamment. Bien

1. L'ayahuasca est une décoction végétale faite à base d'au moins deux plantes: Banisteriopsis caapi et Psichotria viridis.

2. La charte a été précédée par une Assemblée nationale constituante et par plusieurs accords internationaux où se distingue la confirmation de la Convention 169 de l'OIT (1989) sur les peuples indigènes et tribaux, qui reconnaît des droits culturels collectifs à ces populations. 
que les préjugés sociaux de la majorité de la société colombienne sur l'indianité soient toujours présents, on peut affirmer que durant les vingt dernières années, l'identité indigène a acquis une valeur qui était impensable il y a trente ans. La conjoncture suscitée par le multiculturalisme ethniciste adopté par l'État depuis vingt ans a ainsi engendré un terrain propice pour l'urbanisation et l'élitisation de la consommation rituelle du yajé.

La scène sociale de consommation rituelle de yajé est habituellement connue en ville comme prise de yajé. Elle concerne un public hétérogène issu des classes moyenne et haute. Les prises sont des cérémonies privées, rassemblant un nombre restreint de participants et toujours placées sous la direction d'un taita yajecero ${ }^{3}$. Étant donné qu'il s'agit d'une pratique reconnue comme étant d'origine indigène, jusqu'à il $\mathrm{y}$ a peu, ces taitas yajeceros étaient exclusivement indigènes et métis. Ils provenaient de la région du Putumayo-Caquetá, avaient un certain niveau d'initiation chamanique dans l'usage du psychotrope et d'autres plantes fortes, et connaissaient une modalité de travail itinérante entre leur région d'origine et les villes. Quoique, comme nous l'avons déjà dit, la présence de ces spécialistes était courante dans les villes parmi les secteurs populaires - parmi lesquels ils étaient appelés différemment: curanderos, curacas, etc. -, c'est seulement à partir des années 1980 que dans des villes comme Pasto et Bogota, des personnes de classe moyenne intéressées par les savoirs indigènes ont commencé à inviter des taitas yajeceros du Putumayo à offrir des prises de yajé dans des cercles connus de leur propre classe et de la classe supérieure. Professions libérales, intellectuels et artistes trouvent dans les prises de yajé une manière de s'approcher d'une dimension inconnue du monde indigène, hautement valorisée comme ressource thérapeutique face au mal-être du monde urbain.

C'est ainsi que les prises ont commencé à se populariser dans les villes. De ce point de vue, le caractère urbain qu'on attribue à cette nouvelle modalité de la consommation du psychotrope est lié aux attentes de ce nouveau public. Ce sont des facteurs culturels et de classe, propres à ces cercles sociaux, qui rendent « urbaines » certaines prises en usage dans des espaces ruraux (Weiskopf 2002: 115-118). Ces attentes ont aussi provoqué la transformation de certains aspects rituels et discursifs qui caractérisent les prises dans leur modalité traditionnelle. Des réinventions sémantiques et rituelles sont introduites à travers cette nouvelle modalité de la consommation. La principale innovation de cet élargissement du champ yajecero en Colombie consiste en l'apparition d'une nouvelle classe de spécialistes dirigeant des prises de yajé dans les villes: les nouveaux taitas yajeceros.

Ayant été initiés selon les traditions indigènes, ces « chamanes »-comme la plupart d'entre eux se nomment eux-mêmes - établis en ville ont capitalisé la demande urbaine en proposant une pratique rituelle de la prise de yajé qui, sans se défaire de la ritualité

3. La dénomination taita est d'origine quichua, utilisée communément parmi les populations paysannes du sud-ouest du pays pour faire référence aux autorités traditionnelles masculines. Cependant, le terme taita a commencé à être utilisé depuis quelques années pour nommer aussi les spécialistes du yajé. Bien que dans le contexte régional du Putumayo-Caquetá, les spécialistes indigènes du yajé aient été connus sous la dénomination de curacas, cette appellation a cessé d'être utilisée surtout parce qu'elle renvoie à l'idée d'un spécialiste qui maîtrise tant des forces positives de guérison que des forces négatives capables de faire du mal. 
traditionnelle de l'héritage indigène du Putumayo-Caquetá, a intégré de nouveaux langages et de nouvelles propositions adressés à un public majoritairement urbain.

Nous présentons, dans ce qui suit, le cas d'un nouveau taita yajecero établi à Bogota. Bien que ce cas n'épuise pas la variété des manifestations du phénomène, il nous permet d'explorer quelques caractéristiques et traits partagés, à partir desquels on peut différencier des modalités, des accents et des styles dans la consommation rituelle urbaine de yajé en Colombie.

\section{Orlando Gaitan}

J'ai rencontré Orlando Gaitan un matin, dans son bureau de la Fondation Carare dans le nord de Bogota, où je suis arrivée comme n'importe quel patient avec la curiosité de comprendre comment fonctionnait une institution de santé dont le traitement principal était la prise de yajé. Orlando Gaitan est un homme d'âge moyen, grand, à la peau mate et aux cheveux longs. Ce jour-là, il était habillé de blanc; une espèce de serre-tête de billes lui couvrait le front et un collier d'os ajusté décorait son cou. Le bureau était une grande salle décorée de centaines de pièces indigènes venant de toutes les parties du monde. Au début, j'ai remarqué sa disposition à discuter et son amabilité, mais surtout sa manière de parler m'a paru curieuse à cause de fautes grammaticales caractérisées, comme s'il ne connaissait pas correctement l'espagnol. La conversation allant bon train, sa manière de parler se normalisait, laissant entrevoir un homme originaire du nord-est de Colombie qui s'identifiait et se nommait lui-même taita ou médecin traditionnel indigène de l'ethnie carare.

Identifier Orlando Gaitan à cette ethnie est une chose courante dans ces milieux en Colombie. Il est moins connu que les indigènes carare se sont éteints il y a plusieurs décennies. Malgré cela, ce taita est célèbre dans les cercles yajeceros urbains et participe activement à des conférences et à des événements qui traitent du sujet de la médecine traditionnelle indigène. La fondation et le centre médical qu'il dirige comptent des centaines de patients qui assistent régulièrement à des procédures kinésiologiques, qui reçoivent des traitements à base de plantes médicinales et qui participent, bien sûr, à des prises de yajé.

Orlando Gaitan se considère taita, médecin traditionnel indigène ou chamane. Selon son propre récit, son processus d'initiation a été long et varié. Ses débuts remontent à l'époque où il travaillait avec des communautés noires dans la région du Chocó, dans le Pacifique colombien. Il avait décidé de commencer l'apprentissage de la fonction de jaibaná (nom que reçoit le chamane chez les Embera). Il raconte, sans plus de détails, qu'il a voyagé dans la région du Putumayo où il a fait son apprentissage chez des taitas ingas et kofanes des terres basses, mais surtout, chez des taitas siona du fleuve Putumayo. Il mentionne aussi un séjour d'apprentissage sur le fleuve Apaporis (entre le département de l'Amazonie et celui du Vaupés, dans l'Amazonie colombienne). Enfin, il raconte comment, alors qu'il travaillait comme fonctionnaire d'un ministère public, il a connu le légendaire curaca inga Hilario Peña, qui vivait au Guayuyaco dans les terres basses du Caquetá ${ }^{4}$. Il a commencé à prendre de l'ayahuasca et voyageait au départ de la ville chaque fois que cela était possible. Là-bas, dit-il, il a compris que « la médecine

4. Le taita Hilario Peña fut l'un des curacas inga les plus renommés des terres basses du Caquetá jusqu'à sa mort en 1998. 
est une » et que sa mission était d'être un intermédiaire pour amener la médecine à la ville. « Ce furent les anciens qui m'obligèrent à cesser la vie que je menais pour me dédier à celle-ci ». Ainsi termine-t-il rapidement son histoire initiatique. Par la suite, revenir sur le sujet en d'autres conversations fut quasiment impossible. Le silence protège le secret de son pouvoir.

À propos du processus initiatique, toute autre était l'histoire que racontait le taita Antonio Jacanamijoy, aîné inga de Santiago dans la Vallée de Sibundoy, haut Putumayo, avec qui Gaitan a appris les secrets du yajé. Lui et sa famille ont connu Orlando Gaitan au début de l'an 2000. Il est arrivé chez le taita à la recherche d'un traitement pour une maladie grave. Après avoir cohabité durant des mois avec la famille, Gaitan revint à Bogota où, périodiquement, il assistait, comme apprenti et collaborateur du taita, aux prises que celui-ci organisait avec sa clientèle urbaine. Gaitan, de son côté, jouait comme médiateur pour organiser des prises avec d'autres personnes afin que le taita puisse y officier.

À Bogota, Orlando Gaitan a commencé à organiser des prises de yajé en 2003. Bien qu'au début, il légitimât sa pratique comme apprenti du taita Jacanamijoy, en le reconnaissant comme celui qui lui avait donné le pouvoir de guérir, bientôt la référence à son maître disparut. Il se nomma lui-même taita et reprit l'appellation ethnique « carare » comme marqueur d'une ascendance indigène qu'il revendique aujourd'hui et qui le rend légitime face à ses adeptes. Peu de temps après est née la Fondation Carare, une institution dirigée par Gaitan et un groupe de collaborateurs associés, créant un centre alternatif de santé à Bogota. Sous la direction du taita, le centre offre différents services d'assistance médicale, spirituelle et psychologique, selon la politique de la Fondation, inspirée par une « conception holiste de la santé ». On offre aussi des services de consultation, un laboratoire clinique et une pharmacie bio. Une équipe de professionnels en psychologie, médecine et infirmerie, tous apprentis d'Orlando Gaitan, reçoit en moyenne cinquante patients par jour. De nombreux clients de ce centre de santé alternatif appartiennent aux classes moyennes, mais il y a un nombre significatif de personnes des secteurs populaires à la recherche de guérison, ainsi qu'une proportion élevée de femmes. La consultation au sein de ce Centre a un coût moyen de douze euros. À cela, il est coutume d'y ajouter un « apport volontaire » pour le diagnostic du taita. En moyenne, le prix des remèdes d'un traitement simple d'une durée de six mois oscille entre trente et quarante-cinq euros.

Parallèlement au centre, la fondation compte avec « El Sol Naciente », la maloca ${ }^{5}$ construite aux alentours de Bogota, qui fonctionne comme espace rituel pour réaliser les prises de yajé. Les participants à ces cérémonies sont en leur majorité des patients du centre de santé dont le traitement inclut la consommation de yajé. Bien que l'offre de prises de la Fondation Carare soit ouverte au public, pour pouvoir y participer, les personnes intéressées doivent passer préalablement par un diagnostic médical réalisé par les médecins de la Fondation. Les prises de la Fondation se font le week-end,

5. Les malocas sont des constructions inspirées des maisons communautaires de certains peuples indigènes des basses terres, considérées comme des espaces rituels par excellence. Bien que les habitants de la forêt fassent des utilisations très différentes de ces espaces, dans les nouvelles scènes yajeceras, la maloca est conçue comme un espace rituel proprement indigène. En ce sens, nous pouvons dire que les malocas (en tant qu'espace et en tant que dénomination) font partie des marqueurs d'authenticité indigène sur lesquels repose la pratique de la consommation rituelle de yajé. 
et y assistent en moyenne deux cents personnes par session. Le coût est de quinze euros environ, somme couvrant la prise, les bains préparatoires et les "thérapies » ou consultations que dirigent les médecins durant les cérémonies. Ainsi, cette scène yajecero se caractérise par un accent thérapeutique marqué. La Fondation organise aussi des rassemblements et des rencontres entre ses adeptes sur des sujets variés comme les médecines alternatives, l'écologie, le chamanisme, etc. Actuellement, le taita participe activement à diverses activités de la Commission Nationale de Réparation et Réconciliation, instance créée par l'État pour faire le suivi des anciens combattants des groupes paramilitaires réinsérés à la vie civile.

Dans les dernières années, un groupe significatif d'adeptes s'est consolidé autour de la prise de yajé à la Fondation et s'autoproclame la Communauté Carare. Une centaine de personnes compose ce groupe, qui a établi des critères de participation et une forte hiérarchie interne autour d'Orlando Gaitan, son équipe de thérapeutes collaborateurs et leurs adeptes. Les prises de yajé de la Communauté Carare réunissent un grand nombre d'individus et sont caractérisées par un important développement rituel. Aujourd'hui, Orlando Gaitan est l'un des nouveaux taitas les plus connus à Bogota. Il est devenu un véritable leader spirituel pour ses adeptes, qui manifestent envers lui une profonde dévotion.

\section{Nouveaux taitas yajeceros : légitimation et authenticité}

Le cas d'Orlando Gaitan et de la communauté Carare n'est qu'une des modalités actuelles de l'usage rituel de yajé dans les villes: il n'épuise pas la diversité des manifestations de ce phénomène. Il s'agit d'un cas qui révèle certaines tendances, des accents et des styles qui permettent de comprendre les particularités des nouvelles scènes du champ yajecero colombien.

Orlando Gaitan s'autoproclame taita et médecin traditionnel indigène. Pour lui, les deux termes font référence au même concept: il se considère comme un chamane. L'usage du terme « chamane » ne cesse de nous surprendre. En Colombie, à l'intérieur du champ yajecero, ce terme ne s'utilise généralement pas. Sa récente introduction dans certains espaces urbains est liée au champ d'action des nouveaux taitas yajeceros, dont la plupart s'identifient comme tels. Mais que veut dire pour eux être un chamane? D'un côté, les nouveaux taitas comme Orlando Gaitan se voient eux-mêmes comme des spécialistes héritiers d'une tradition indigène (la tradition yajecera) : c'est pourquoi ils se nomment eux-mêmes taitas. De l'autre, on observe ces dernières années que les nouveaux yajeceros utilisent la littérature anthropologique comme source de légitimation, proposant une assimilation entre la figure du taita et la catégorie de chamane $^{6}$. Ce n'est pas une coïncidence si bon nombre de ces nouveaux taitas ont une formation universitaire en anthropologie ${ }^{7}$. Ainsi, les nouveaux taitas se voient comme des « médecins traditionnels », bien qu'ils soient différents des taitas yajeceros traditionnels

6. Il est nécessaire de remarquer que durant ces vingt dernières années en Colombie, l'introduction de l'industrie éditoriale New Age a été vertigineuse. Ce type d'industrie culturelle met un accent tout particulier sur les techniques de dépassement personnel dérivées de multiples modèles thérapeutiques alternatifs.

7. La transformation idéologique de ce nouveau chamanisme doit beaucoup au rôle des anthropologues adeptes du rituel, qui font un usage intensif du savoir expert pour légitimer la pratique. 
(auprès desquels ils se sont initiés); leur position dans cette conjoncture historique d'expansion de la médecine traditionnelle indigène est celle d'intermédiaires entre le monde indigène et le monde urbain à travers les processus de guérison. Leur autoassignation à la catégorie de chamane tient à ce qu'ils se considèrent comme des spécialistes polyvalents ayant la capacité de passeur à travers l'incommensurabilité de cultures différentes. Les nouveaux taitas se voient donc avant tout comme des intermédiaires, des liens entre des cultures; leur pouvoir réside dans leur capacité à juxtaposer divers registres. À l'intersection des cultures, ils se considèrent comme des traducteurs ayant la capacité de guider les expériences individuelles des adeptes urbains du yajé à partir de plusieurs modalités de chamanisme - et non seulement à partir de celle du yajecero. Ainsi, au sein de ce champ, c'est surtout le type d'articulation qu'ils établissent entre la «tradition » et une demande urbaine croissante qui les différencie d'autres modalités yajeceras, et qui leur permet une flexibilité et une polyvalence à partir desquelles ils ont introduit des adaptations et des réinventions au niveau du rituel, du langage, des sens, etc.

Tout comme Orlando Gaitan, la plupart des nouveau taitas assurent répondre à un appel que les vieux taitas leur ont fait: ils les ont " élus » comme personnes-clés pour ouvrir les portes des « savoirs indigènes " aux non-indigènes. C'est pourquoi l' « élection » par ces mêmes aînés apparaît fondamentale comme mécanisme de légitimation. Comme marqueur d'authenticité, l'" élection " attribuée aux taitas maîtres positionne le nouveau taita comme continuateur de la tradition indigène ${ }^{8}$. Cette élection sert en même temps à légitimer la condition de pont ou de médiateur entre deux mondes, ce qui est généralement revendiqué comme une « mission » de la part des nouveaux taitas.

Si pendant plus d'une décennie, ce sont les intellectuels et les universitaires de la ville qui ont soutenu et légitimé la pratique des taitas du Putumayo en ville, il faut remarquer que, récemment, l'équation s'est inversée. Ces dernières années, ce sont principalement les taitas aînés (la plupart indigènes), chantres de l'urbanisation de la « médecine traditionnelle » durant les années $1990^{9}$, de même que les représentants politiques indigènes autorisés par l'État, qui servent de sources de légitimation de la pratique des nouveaux taitas urbains. Ce fait est significatif pour comprendre l'usage actuel du yajé dans les villes colombiennes.

Dans la tradition yajecera, un apprenti reçoit la couronne de plumes de guacamaya ${ }^{10}$ ou la waira $^{11}$ des mains de son maître comme symbole de reconnaissance de son

8. Bien que la plupart de ces nouveaux taitas fussent initiés pendant des périodes beaucoup plus courtes que celles d'application pour leurs maîtres, le fait d'avoir intégré les conditions de vie autochtones devient, bien souvent, suffisant pour légitimer cet apprentissage. Le déplacement vers la « jungle » - ou vers les territoires indiens - masque le temps réduit de préparation. La distance spatiale se superpose à la distance temporelle.

9. C'est le cas de la génération des yajeceros célèbres qui rendirent visible le yajé dans les villes dans les années 1990. Il s'agit de quelques anciens reconnus des groupes indigènes du Putumayo, les premiers à sortir en ville avec l'aval de spécialistes non indigènes. Ils figurèrent dans les médias nationaux et internationaux, et furent les informateurs de la plupart des travaux récents sur ce sujet.

10. Grand perroquet coloré vivant dans les forêts tropicales d'Amérique du Sud.

11. La waira est le balai rituel utilisé par les yajeceros pour balayer ou laver les entités négatives dans des objets, un espace ou des personnes. 
pouvoir de guérison donné par les esprits auxiliaires qui habitent le monde du yajé. Or ici, selon l'un des certificats exposés sur un mur de la Fondation, Orlando Gaitan a reçu la couronne qui symbolise la reconnaissance d'un taita yajecero de la part de l'autorité indigène du cabildo $^{12}$, reconnue par l'État, qui lui concède le pouvoir comme taita sous forme d'un certificat légal. Ce type de superposition entre autorité, authenticité, et marque ethnique de légitimité montre bien les enjeux des représentations de l'indianité, qui servent comme source de légitimité des nouvelles modalités urbaines de consommation de yajé.

Néanmoins, au-delà de cela, Orlando Gaitan ne légitime pas seulement son lien avec la tradition yajecera par son apprentissage. En s'autoproclamant héritier direct de la tradition indigène carare, il fait de la marque ethnique un fondement plus profond de l'autorité et de l'authenticité indigènes, en introduisant comme variable la continuité du lien de sang ${ }^{13}$.

Comme figures d'intersection, les propositions des nouveaux taitas yajeceros pour leur public urbain croissant sont souvent caractérisées par une empreinte personnelle selon l'accent qu'ils donnent à la consommation rituelle de yajé, et selon le style propre de chacun. Ruptures, continuités et innovations du dispositif rituel des prises de yajé accompagnent les nouvelles représentations du pouvoir de ce psychotrope construites par les nouveaux consommateurs.

\section{Médicalisation des prises de yajé}

L'un des changements les plus substantiels dans la manière dont les gens de la ville se représentent la consommation rituelle de yajé tient dans la vision qu'ils s'en font comme « médecine traditionnelle indigène ». Il ne s'agit pas seulement d'une manœuvre publicitaire de ceux qui offrent du yajé dans les villes, mais de l'élaboration d'un discours, plus ou moins institutionnalisé et utilisé par différents acteurs au sein du champ yajecero, comme stratégie pour augmenter leur visibilité et leur légitimité. Présenter la consommation rituelle de yajé comme une pratique typique de la tradition médicale indigène assure aux yajeceros une certaine reconnaissance, qui leur donne la possibilité de maintenir leur pratique urbaine au sein de la légalité.

Bien que l'appellation de " médecine traditionnelle indigène » puisse paraître indiscutable pour beaucoup, le fait que son usage soit récent permet de suivre à la trace les transformations du sens que les gens de la ville ont donné à cette notion. De nos jours, l'idée de « médecine traditionnelle » (MT) est communément admise par tout citadin, mais il n'en a pas toujours été ainsi. Selon des auteurs comme Javier Lozoya pour le cas latino-américain, la notion de MT a commencé à circuler largement au plan mondial à partir des années 1970, grâce à un mouvement intellectuel qui, inspiré par la stratégie de santé développée en Chine, proposait la revalorisation de pratiques curatives des cultures médicales restées en dehors du développement scientifique et

12. Bien qu'à l'origine le cabildo fût une institution coloniale, depuis la république, et notamment après la nouvelle constitution, il est devenu le représentant de l'autorité traditionnelle indigène reconnue par l'État pour chaque groupe ethnique.

13. Dans le champ traditionnel, un yajecero n'obtient pas seulement son pouvoir par l'apprentissage. Les représentations sur le sang indien comme source naturelle de pouvoir sont aujourd'hui sanctionnées par la marque ethnique (voir Pinzón, Suarez et Garay 2003 : 166-168). 
technologique occidental (Lozoya 1993 : 111). Repris par l'Organisation mondiale de la santé(OMS), le «modèle chinois » représentait une stratégie intéressante de combinaison de savoirs médicaux qui pouvait s'appliquer aux pays en développement. Mais c'est à partir de la conférence d'Alma Ata - convoquée par l'OMS et l'UNICEF en 1978 - que s'est instauré formellement le « programme de promotion et de développement des médecines traditionnelles » (créé par l'OMS en 1975) comme politique de soins de santé primaires dans les pays du Tiers-Monde. Ainsi, la MT apparaît comme une alliée de la médecine scientifique; l'articulation entre les systèmes médicaux académiques et traditionnels devient une stratégie pour l'amélioration de la couverture sanitaire dans les pays pauvres, là où les services généraux de santé sont déficients (Lozoya 1993: 112).

En Colombie, les notions de «médecine traditionnelle » et de «chamanisme » se sont déployées à l'origine principalement dans les milieux universitaires, en particulier au départ de travaux d'anthropologues qui ont étudié les systèmes de santé et de maladie dans des contextes socioculturels particuliers. À partir des années 1990, les deux concepts ont commencé à circuler plus largement en dehors des milieux universitaires, pour faire référence à des pratiques thérapeutiques présentées comme appartenant au monde indigène. L'étiquette de Médecine Traditionnelle Indigène est liée à la façon dont cette pratique a commencé à circuler dans certains milieux urbains comme une forme de promotion des prises de yajé organisées par des intermédiaires qui invitaient des taitas yajeceros du Putumayo à la ville.

Cependant, l'introduction du nom de MTI n'aurait pas été aussi efficace si elle n'avait pas été réalisée dans un contexte favorisé par la décentralisation administrative de l'État et la restructuration du système national de santé selon la loi $100^{14}$. Le nom de MTI a acquis une légitimité en tant qu'élément fondamental des politiques différentielles de santé reconnues pour les populations ethniquement marquées. Parallèlement, l'intervention d'agences de coopération et d'organisations non gouvernementales dans ce domaine favorise la revitalisation de la MTI à la lumière des orientations de politiques sanitaires établies par des organisations internationales comme l'OMS. À cet égard, ce n'est pas un fait négligeable que l'OMS établisse une articulation entre la MT et ce qu'on appelle les Médecines Complémentaires ou Alternatives (OMS 2002: 1-6).

Depuis le milieu des années 1990, les nouveaux yajeceros indiens et métis ont recours à cette dénomination selon une stratégie visant à installer leur pratique dans le champ de plus en plus étendu des services complémentaires et alternatifs de santé. Ainsi, l'usage rituel de yajé entre dans le marché des alternatives médicales et thérapeutiques, où il se trouve en concurrence avec (entre autres) l'homéopathie, l'acupuncture, la médecine chinoise, la Gestalt, la biodanse, l'aromathérapie, le yoga, etc. Mais en même temps, l'usage du yajé s'est complété avec ces soins alternatifs. Au sein de ce spectre, l'offre est large et les nouveaux taitas yajeceros jouissent d'une certaine souplesse dans leur pratique grâce à leur capacité d'articuler l'usage du yajé avec d'autres techniques, langages et rituels venant des systèmes médicaux et thérapeutiques alternatifs.

Comme on le voit, le processus de légitimation des nouveaux yajeceros urbains est fondé en partie sur l'accent mis sur le caractère indigène, réévalué et reconnu comme un marqueur de diversité culturelle et d'inclusion. Les représentations qui valorisent

14. Cette loi est celle qui organise en Colombie la restructuration du Système National de Santé en privatisant la sécurité sociale. 
l'exotisme de la différence ethnique et culturelle sanctionnent aussi les transformations de ce qui était auparavant considéré comme croyance et qui maintenant est apprécié comme connaissance. Bien sûr, ce changement des représentations a été rendu possible par la médiation de secteurs intellectuels et académiques comme source de légitimation sociale.

Cependant, un autre aspect du processus de légitimation de ces pratiques est lié à leur considération comme pratiques «médicales ». Dans ce contexte, on est frappé par l'usage régulier d'une terminologie médicale de plus en plus standardisée au sein du champ yajecero. Aujourd'hui, sur la plupart de ces scènes, il est courant de parler de « séance », « traitement », « thérapie », « dose », « cure », « diagnostic », « médecine », « patient », « pathologie », « histoire clinique ». Ainsi, il est possible de trouver des lieux comme celui de la Fondation Carare, où la consommation de yajé a pour condition que le « patient» dispose d'une « histoire clinique » à partir de laquelle certaines « maladies » sont « diagnostiquées » et des « thérapies » sont « prescrites », parmi lesquelles la prise de «médicaments » comme le yajé. Bien que la médicalisation puisse être considérée comme le résultat de circonstances contingentes, il faut garder à l'esprit que cette mise en avant de la dimension thérapeutique a été récurrente dans d'autres moments historiques et dans d'autres contextes. Comme Roberte Hamayon et Jean Langdon le rappellent, la valorisation historique de la composante thérapeutique est liée aux stratégies adaptatives déployées dans des situations de colonisation. (Hamayon 2003 : 26 et Langdon 1991 : 57). Néanmoins, pour notre cas, cela a plusieurs implications.

Il y a d'abord une tendance à assimiler les pratiques thérapeutiques associées au yajé avec la médecine allopathique. Cela signifie qu'on tend à comprendre la pratique des taitas avec une logique et une structure comparables à celles de la science médicale occidentale. L'équivalence n'est pas totalement exacte. L'analogie avec la médecine occidentale focalise la pratique thérapeutique autour d'une seule personne: le spécialiste. On tend facilement à confondre la pratique du taita yajecero avec l'idée $\mathrm{du}$ « médecin indigène » ou du « médecin des Indiens ». Dans ce contexte, on ignore non seulement le caractère multidimensionnel de la pratique des yajeceros, mais aussi l'éventail des spécialisations existant au sein des chamanismes yajeceros indiens et métis. La tendance à l'individualisation du statut de spécialiste se manifeste dans la relation croissante de taitas reconnus avec la population métisse et urbaine, où se trouve la plupart de leur clientèle. À leur tour, ces connaissances deviennent l'apanage de moins en moins de personnes et sont alors simplifiées. Ainsi, la prétention de faire correspondre le rôle du médecin occidental avec celui du taita yajecero n'est possible qu'à condition de simplifier et standardiser le champ pratique de ce dernier. Selon Jean Langdon, il se produit dans ces cas une sorte de caricature du rôle du taita yajecero (2007: 115).

Bien que la MTI soit née, en tant que terme anthropologique et politique, pour définir la « médecine des indigènes » dans les années 1970, cette notion a été progressivement réappropriée dans un sens de légitimation des tentatives des nouveaux yajeceros pour créer des modèles thérapeutiques alternatifs, dans une tentative de donner une légitimité scientifique à leur pratique. En termes comparatifs, il est intéressant de voir que dans le cas colombien, l'usage médical du yajé semble être la voie la plus rapide de sa légalisation et de sa légitimation, alors que dans le cas brésilien, cet usage médical est interdit tandis que son usage religieux est autorisé. 


\section{Le modèle thérapeutique et le lieu du féminin}

En tant que centre de soins de santé, la Fondation Carare a un schéma général de fonctionnement pour le diagnostic de sa clientèle. Chaque nouveau patient doit fournir les données nécessaires de son histoire clinique. À partir de là, il ira en consultation avec un médecin de garde qui fera une estimation générale du cas, établira un diagnostic puis remettra le patient au taita pour l'approbation du traitement et la prescription de médicaments à base de plantes. Les consultations de la Fondation Carare sont d'habitude centrées sur deux éléments: d'une part, l'histoire de vie du patient; d'autre part, une lecture du corps qui permet de définir certains indicateurs, ce qui fait partie du modèle de diagnostic de la Fondation. En effet, selon Orlando Gaitan, le corps constitue un texte où il est possible de lire la nature du problème qui produit la maladie chez le patient. Dans cette perspective, la lecture du corps dans son ensemble et dans ses parties (des asymétries, des douleurs, des tensions, des faiblesses, etc.) permet de déterminer la cause ultime du malaise, lequel, bien qu'ayant une manifestation physique, renvoie à un désordre de caractère comportemental, c'est-à-dire qu'il renvoie au domaine des relations sociales que la personne noue dans sa vie quotidienne ${ }^{15}$. Le fait que la maladie soit considérée avant tout comme la conséquence d'un mauvais comportement ou, comme le prétendent les adeptes, d'un « ressentir mal géré », renvoie à une interprétation qui définit le processus de guérison en termes moraux. En d'autres termes, la guérison est avant tout un exercice moralisant.

Ce modèle de diagnostic est la spécialité du taita: aussi, les cas qui arrivent aux médecins sont passés en revue par lui pour établir le traitement à suivre. Les traitements de la fondation comprennent deux modalités: d'une part les thérapies et les remèdes; d'autre part, les prises de yajé. Parmi les thérapies offertes par le centre, on distingue les « thérapies du dos » (qui consistent en des séances de massage), les « thérapies d'estomac », les «thérapies de sein », les "thérapies d'utérus », etc.; il y a aussi les bains, l'encens et les dépouillements - c'est-à-dire des séances finales de propreté réalisées à la fin d'un traitement. En ce qui concerne les remèdes, il s'agit pour la plupart de sirops à base de plantes médicinales mis en vente au même endroit. Quant aux prises de yajé, elles sont prescrites comme faisant partie des traitements médicaux. On considère que le yajé permet de "voir » la cause ultime de la maladie (le yajé montre quand et comment le mauvais comportement a eu lieu) et permet le nettoyage qui rendra la guérison possible. Les prises sont réalisées dans la maloca construite pour cela. Là aussi, une procédure a été établie à l'attention des participants.

Les séances de la Communauté Carare ${ }^{16}$ intègrent les patients du centre et les preneurs réguliers de la communauté. Celui qui assiste pour la première fois à une prise dans cette maloca doit passer par une préparation et prendre un bain préalable avant d'entrer. De même, il doit avoir une consultation avec les médecins préalablement à la prise, puisque, avant d'initier la cérémonie, le taita doit connaître le cas des novices pour pouvoir orienter la guérison par le yajé. Les nouveaux preneurs recevront au cours de la séance la thérapie prescrite. Ils seront finalement impliqués dans la partie de la

15. Il est à noter que l'une des maladies les plus diagnostiquées dans le centre est le «stress » lié au mode de vie. Selon Orlando Gaitan, le stress est dû au désordre, produit de la colère, qui provoque la peur (notes de terrain, janvier 2009).

16. Nom adopté par les adeptes du taita Orlando Gaitan et de sa Fondation. 
séance consacrée aux guérisons ${ }^{17}$. Administrées directement par le taita et ses assistants masculins, les guérisons consistent en séances individuelles de nettoyage au moyen du chant, de la waira, d'orties et d'autres éléments rituels par lesquels la maladie est retirée du corps. Les traitements offerts par la Fondation consistent en moyenne en six séances de thérapies et en au moins deux prises de yajé.

Un bon exemple de la façon dont les yajeceros urbains combinent la tradition indigène du yajé ${ }^{18}$ avec des innovations adaptées aux attentes du public urbain est le modèle thérapeutique destiné à la clientèle féminine. Comme nous le remarquions plus haut, la Fondation reçoit un nombre important de patients de sexe féminin. Le fait que les médicaments complémentaires et parallèles aient plus de succès avec les femmes a été documenté par plusieurs chercheurs, bien qu'il semble difficile de faire des généralisations sur les raisons de ce fait. Bien que de nombreuses femmes viennent pour des problèmes physiques, beaucoup d'autres y vont par recommandation d'amis et de parents, à la recherche d'un remède aux affections relatives aux problèmes de travail, de famille, de couple, d'estime de soi, de dépression, de perte, de violence physique, de maltraitance, etc. Face à cette demande variée, il est intéressant de remarquer que les problèmes associés à l'utérus s'avèrent les diagnostics les plus fréquents. Par l'analyse d'échantillons médicaux dans le laboratoire, le taita détermine l'état de l'utérus de la patiente, et définit le type de traitement à suivre. Les traitements visent à "nettoyer » l'appareil reproducteur féminin au moyen de certaines thérapies qui incluent des bains de siège, de l'encens et des sirops médicinaux, mais aussi en établissant de nouvelles normes de comportement moral.

Selon Gaitan, l'appareil reproducteur féminin, en tant que créateur de vie, fait que la femme a plus de composantes du corps que l'homme. Cette théorie du corps féminin se fonde sur une conception différentielle des forces féminines et masculines du monde: dans le cas de la femme, cette théorie est déterminée par sa capacité de concevoir et par le pouvoir attribué à son cycle. En raison de sa condition (plus de composantes corporelles), la femme aurait plus d'émotivité que l'homme et serait donc plus en mesure de faire des erreurs (comportement social), ce qui la rend plus sensible à la maladie. La théorie qui soutient ces représentations indique que le cycle menstruel est un moment où l'utérus est ouvert pour permettre l'expulsion du sang menstruel. Ce dernier est considéré comme une source importante de contamination puisque par celuici est expulsée la charge énergétique négative des relations sociales que la personne a vécues pendant les vingt-huit jours du cycle. En ce sens, les diagnostics sur l'état de l'utérus permettent de déterminer la cause de l'affection, et les thérapies sur l'utérus permettent de nettoyer les effets de l'émotivité accrue de la femme, la prise du yajé rendant possible de réévaluer ses règles de comportement pour obtenir une guérison physique, psychologique et spirituelle.

17. Les prises de yajé sont composées d'au moins trois moments : la préparation, où le yajé est consacré et réparti; la prise, le moment où les participants expérimentent les effets les plus forts; et la fermeture, le moment des guérisons.

18. L'aspect le plus évident de ce qu'on appelle ici la tradition indigène yajecera est le dispositif rituel de la prise de yajé. La structure et l'ordre que le rituel de prise de yajé impose dans ces nouvelles scènes sont considérés comme hérités directement de la tradition indigène. C'est pourquoi les règles régissant le processus rituel apparaissent comme si strictes et peu flexibles dans certains cas. 
Bien que l'accent mis sur le cas des femmes soit un point fort du centre, le modèle thérapeutique du centre ne se réduit pas à ce seul genre de traitement. Les représentations qui soutiennent le modèle thérapeutique engagent des processus de resignification d'aspects fondamentaux du chamanisme yajecero traditionnel, tels que l'exclusion des femmes et le tabou du sang menstruel ${ }^{19}$. Au sein du champ yajecero traditionnel, le sang menstruel est opposé au pouvoir de guérison du yajé. Le sang menstruel endommage la puissance d'un taita, peut le rendre malade et même le tuer, et a le pouvoir d'abîmer le yajé ${ }^{20}$. De même, une femme qui vient d'accoucher est considérée comme dangereuse puisque son utérus reste ouvert pendant un certain temps ${ }^{21}$. C'est pourquoi l'on exclut les femmes ayant leurs règles et les femmes venant d'accoucher des espaces de préparation ou des rituels et, en général, des espaces des taitas.

En milieu urbain, ces logiques apprises par les nouveaux taitas dans leur processus initiatique sont actualisées et re-signifiées. Dans le cas de la Fondation Carare, les restrictions d'accès des femmes sont respectées de façon stricte et sont légitimées en tant que conditions propres à la tradition indigène. C'est pourquoi les espaces du Centre ainsi que la maloca sont interdits aux femmes pendant le cycle menstruel (même pour l'équipe médicale). La restriction opère par le biais de plusieurs mécanismes de régulation de l'accès: ainsi, dans le cadre des préparatifs de la cérémonie, on oblige les participantes à faire des bains de siège pour nettoyer le vagin. En ce qui concerne Orlando Gaitan, ses adeptes ont établi des réglementations spéciales de soins et de protection non seulement contre les risques liés à une femme ayant ses règles, mais contre tous les facteurs qui pourraient abîmer le pouvoir de guérison de leur chef ${ }^{22}$.

\section{Quelques réflexions finales}

L'expansion contemporaine du champ yajecero en Colombie se manifeste par l'urbanisation des prises de yajé et par son appropriation par une élite. Cette nouvelle et croissante modalité urbaine a acquis de la légitimité (et par là même, de la légalité) grâce à l'adoption de discours experts (anthropologiques et médicaux, principalement) et grâce aux politiques multiculturelles promues par l'État colombien. Le cas de la Fondation Carare est un exemple intéressant de la manière dont les représentations

19. Cette conception est fondée sur une idée commune aux peuples indigènes du piémont amazonien colombien, à savoir que les femmes ont la capacité d'attirer les maladies (des entités négatives ou des esprits) qui se déchargent périodiquement par la menstruation. « Les femmes ont un pouvoir au moins similaire à celui des chamanes, qui ne dépend pas d'un entraînement comme celui qu'ils doivent suivre pour la manipulation de ces forces. Bien qu'il soit certain qu'elles n'ont pas le pouvoir d'enlever les maux, elles peuvent les recueillir, les acheminer et les décharger » (Amaya $2008: 4-5$ ).

20. Au sein du champ traditionnel yajecero indigène et métis, le yajé est considéré comme " jaloux » et doit être protégé de la vue du public où il y a généralement des femmes. On croit que le regard d'une femme ayant ses règles peut rendre complètement inopérante la préparation.

21. Parmi les populations indigènes de Colombie, l'homme dont la femme vient d'avoir un bébé est aussi exclu des espaces du yajé pendant la période post-partum.

22. La communauté Carare a ainsi mis en place en son sein un ordre hiérarchisé entre ses membres, qui fonctionne comme des cercles concentriques de protection autour d'Orlando Gaitan. Ces cercles accomplissent des fonctions logistiques au sein des activités de la Fondation mais aussi des fonctions spécifiques à l'intérieur du rituel. 
de l'authenticité indigène se combinent à partir de certaines représentations de la continuité de la tradition indigène, et de pratiques qui s'en détachent. La place des femmes dans la tradition yajecera est mise à jour et re-signifiée, en devenant un point fort de la proposition thérapeutique urbaine et un tremplin pour gagner de la clientèle. Cependant, l'univers des significations liées aux attentes du nouveau public qui accède à ces espaces entraîne une réélaboration permanente des propositions et des styles des nouveaux taitas.

La Fondation offre un cadre qui prête une attention particulière au bien-être individuel, tout en proposant de plus un glissement de l'idée de guérison du physique au spirituel : ceci explique sans doute son succès auprès des femmes, qui cherchent une résolution à leurs drames personnels. En outre, les femmes trouvent dans ce modèle thérapeutique une possibilité de prise en charge où l'on reconnaît leur genre comme un marqueur différentiel qui définit des règles spatiales de soins. Cette valorisation du féminin n'est pas une exclusivité de la Fondation: dans de nombreux milieux urbains, la condition féminine acquiert une importance centrale dans les discours et les pratiques des yajeceros. Étant donné qu'au sein du champ traditionnel, le yajé est associé aux pouvoirs des hommes, ces nouveaux espaces urbains (qui se considèrent des héritiers de la tradition yajecera) adoptent des mécanismes et des stratégies d'inclusion des femmes tant au niveau discursif que pratique, en renouvelant les modes de consommation du psychotrope.

Aux yeux des yajeceros urbains, le «pouvoir de la femme » est généralement associé à certaines valeurs sacrées liées à la nature, à la maternité et à la menstruation, valeurs jugées vitales mais sous-estimées par la société techno-industrielle. Ainsi, plusieurs des nouvelles scènes de prise de yajé ont adopté des rituels complémentaires aux prises que l'on présente comme des cérémonies associées au pouvoir des femmes. C'est le cas du inipi (sweatlodge), rituel dont l'origine est censée être lakota et qui est devenu chose courante dans de nombreux milieux yajeceros urbains (Caicedo 2009:22-25). Sans doute ce genre d'innovation se comprend-t-il mieux si l'on considère que les consommateurs urbains de yajé ont l'habitude de partager leurs univers de significations avec des adeptes d'autres alternatives thérapeutiques, spirituelles et rituelles associées au vaste champ sémantique du New Age et de son industrie culturelle.

La tendance croissante à donner de la visibilité au féminin peut être considérée comme une innovation propre à l'expansion du champ yajecero urbain. Le processus d'urbanisation des prises et son appropriation par des élites ont mis en évidence le caractère masculin de la tradition yajecera, tout en ouvrant en même temps un espace pour compenser le déséquilibre à travers la participation des hommes et des femmes dans ce type de pratique. En considérant le milieu urbain et les classes sociales impliquées, il aurait été très difficile de maintenir ce déséquilibre. Ces espaces ou sphères complémentaires pour les femmes, innovation des nouveaux taitas yajeceros, gagnent progressivement en visibilité auprès du public urbain.

En Colombie, la croissance de la consommation rituelle de yajé a été rendue possible grâce au contexte multiculturel: l'État a fait de l'indianité une source de légitimité. Le processus d'urbanisation et d'élitisation favorise les individus capables d'organiser l'usage traditionnel du psychotrope (reconnu comme autochtone) en fonction des attentes du public urbain. Dans les villes, la pratique des taitas est institutionnalisée sous le nom de "médecine traditionnelle indigène ", en usant de références qui présentent les prises comme de nouvelles formes de thérapie. En utilisant 
une terminologie de plus en plus médicalisée, les nouveaux yajeceros adaptent leurs pratiques aux modèles thérapeutiques alternatifs. En résumé, l'utilisation thérapeutique du yajé prospère dans la mesure où elle est simplifiée et standardisée comme un service thérapeutique alternatif. Le vaste champ des nouveaux usages du yajé ouvre ainsi un jeu de significations et d'investissements dont l'analyse anthropologique est intéressante : la présente contribution n'avait d'autre ambition que d'en suggérer quelques explorations possibles.

\section{Références citées}

AmaYa, Carolina, 2008. « Medicina Tradicional Indígena: Opción saludable para la mujer ». (Fascículo 15, Grupo de estudios en sistemas tradicionales de salud - GESTS). Bogota: Universidad del Rosario.

CAicedo, Alhena, 2009. « Nuevos chamanismos, Nueva Era », Revista Universitas Humanistica, 68: 15-32.

-, 2010. «El uso ritual del yajé. Patrimonialización y consumo en debate », Revista Colombiana de Antropología, 46 (1): 63-86

Hamayon, Roberte, 2003. « Introduction à Chamanismes. Réalités autochtones, réinventions occidentales », in Roberte Hamayon (dir.), Chamanismes, 7-54. Paris : Presses Universitaires de France.

LANGDON, Esther Jean, 1991. «Interethnic processes affecting the survival of shamans: a comparative analysis », Otra América en construcción. 46 Congreso Internacional de Americanistas. Memorias del simposio Identidad cultural, medicina tradicional y religiones populares. Universidad de Ámsterdam 1988, 44-65. Bogota : Instituto Colombiano de Antropología - ICAN.

-, 2007. « Problematizando os projetos de Medicina Tradicional Indígena », Medicina Tradicional Indígena em contextos. Anais da I Reuniao de Monitoramento. Area de Medicina Tradicional Indígena Projeto Vigisus II/ Funasa, 110-119. Brasilia : Ministerio da Saude.

Lozoya, Xavier, 1993. « La medicina tradicional en México en la perspectiva de fin de siglo », in Carlos Pinzón, Rosa Suarez et Gloria Garay (dir.), Cultura y salud en la construcción de las Américas. Reflexiones sobre el sujeto social, 109-117. Bogota: Instituto Colombiano de Antropología - ICAN.

OMS, 2002. Stratégie de l'OMS pour la Médecine Traditionnelle pour 2002-2005. Genève: Organisation Mondiale de la Santé.

Pinzón, Carlos, Rosa SuÁrez et Gloria Garay, 2003. Antropología de la salud. Itinerario autoetnográfico por el poder mágico y la curación. Bogota : Universidad Nacional de Colombia.

WeISKopf, Jimmy. 2002. Yajé. El nuevo purgatorio. Bogota : Villegas Editores. 